\title{
La formación humana y la universidad pública: algunas contradicciones
}

\author{
Human education and the public university: many contradictions
}

DOI 10.15517/rr.v99i1.38430

Sonia Angulo Brenes ${ }^{1}$

${ }^{1}$ Escuela de Trabajo Social, Universidad de Costa Rica, Costa Rica, sanbre.05@gmail.com

Fecha de recepción: 24 de julio de 2019

Fecha de aceptación: 17 de enero de 2020

\section{Resumen:}

El objetivo del artículo es discutir las relaciones y las contradicciones entre la formación humana y la educación en la universidad pública, esto con la finalidad de comprender su reproducción en la sociedad capitalista. La formación humana se expresa a partir de la posibilidad de que, el género humano como ser social, se configure a través del trabajo como categoría fundante, mientras que la función de la educación se impone principalmente en el desarrollo de la sociedad capitalista donde, producto de esta contradicción, se produce una fractura en este ser social, pues esta formación se convierte en preparación para el trabajo. De ahí deviene la necesidad de la creación de diversas instancias para cumplir tal finalidad, una de ellas es la universidad pública como espacio contradictorio ya que, por un lado, su finalidad es la formación para el cumplimiento de un mercado de trabajo; pero por el otro, permite la adquisición de conocimientos universales que proporciona al colectivo social apropiarse y divulgar esa comprensión para su reproducción como sociedad. Una de las principales conclusiones es la necesidad como sociedad de entender estas contradicciones y su función social en la creación y colectivización del conocimiento.

Palabras claves: Capitalismo, género humano, empleo, conocimiento; mercado de trabajo.

Abstract: The objective of this paper is to discuss the connections and the contradictions
between human background and education in the public university to understand its
development in the capitalist society. The human background is showed from the possibility
that the humankind like a social being can be configured through the work like a basic
category, while the role of the education imposes itself mainly against the capitalist society,
where as a result of this contradiction it makes a rupture in this social being, so this education
transfigures in preparation for the work. Hence, it comes about the necessity of the creation
of several instances to accomplish this purpose; one of them is the public university as a (i) (1) $\Theta \Theta$

Este obra está bajo una licencia de Creative Commons Reconocimiento-NoComercial-SinObraDerivada 4.0 Internacional. 
contradictory space, by one side its objective is the background to satisfy a work market and by the other side, it allows the acquisition of universal knowledge that provides the social collective to take and reveal this basic comprehension for its development as a society. One of the mainly conclusions is the need as society of understanding these contradictions and its social roles in the creation and collectivization of the knowledge.

Keywords: Capitalism, mankind, work, knowledge, work market.

\section{Introducción}

Uno de los elementos fundamentales para comprender las formas de reproducción de la sociedad capitalista, es la contradicción entre la formación humana (como proceso de adquisición universal de conocimiento) y la académica (como una preparación para el trabajo) en el contexto de la universidad pública. A partir de esta paradoja, surgen las siguientes dos preguntas: ¿cómo entender la fractura del género humano entre un conocimiento universal frente a uno atomizado? Y ¿cómo pensar las posibilidades de la formación académica desde la educación superior pública ante estas contradicciones? ${ }^{1}$.

De ahí que la propuesta analítica para discutir esta relación, entre formación humana y académica, parte de una comprensión ontológica, en la cual, la constitución del género humano se basa en su reproducción como ser social a partir del trabajo como categoría fundante. En este sentido, Marx $(2010,185)$ explica que es «un proceso entre el hombre y la naturaleza, proceso en que el hombre realiza, regula y controla, mediante su propia acción, su intercambio orgánico con la naturaleza. El hombre se enfrenta a la materia natural como una potencia de la naturaleza. Pone en movimiento las fuerzas naturales que pertenecen a su corporeidad, los brazos y las piernas, la cabeza y la mano, para apropiarse de la materia

\footnotetext{
${ }^{1}$ Estos cuestionamientos surgieron a partir de la reflexión de la formación académica en la Escuela de Trabajo Social de la Universidad de Costa Rica como producto de un análisis sobre lo que se llama en la profesión los fundamentos del Trabajo Social, en el marco del proyecto de investigación Cuestión Social, Fundamentos del Trabajo Social y tradición marxista en la Escuela de Trabajo Social de la Universidad de Costa Rica 20042016. (c) (1) (8) $\Theta$
} 
natural en una forma útil para su propia vida. Al actuar por medio de este movimiento sobre la naturaleza exterior y transformarla, modifica a la par su propia naturaleza».

A partir del trabajo, el ser humano, en su relación constante con la naturaleza, la transforma y al mismo tiempo, se transforma a sí mismo. Es en este vínculo que se genera la formación humana, estableciendo las bases históricas para la reproducción de la sociedad y permitiendo el constante desarrollo de los conocimientos de la colectividad. Sin embargo, principalmente con la instauración de la sociedad capitalista, se produce una fractura de ese ser social por la contradicción entre el desarrollo del trabajo frente al dominio del capital.

La sociedad está subordinada a la división del trabajo y, por tanto, la contradicción capital-trabajo enfrenta cada día más una agudización en sus expresiones en la cotidianidad, «el sistema del capital es, en realidad, el primero en la historia que se constituye como totalizador innegable e irresistible, no importa cuán represiva deber ser la imposición de su función totalizadora en cualquier momento y en cualquier lugar en que encuentre resistencia» (Mészáros 2002,97). A partir de esta contradicción, la formación humana sufre una fractura, su finalidad se convierte en la preparación para el trabajo y, para cumplir esta función, empiezan a aparecer entidades con esta finalidad. Una de ellas es la universidad pública que, al mismo tiempo que provee conocimientos universales, prepara para el trabajo. En este sentido, cuando se analizan las posibilidades de la formación académica y su relación con el conocimiento universal, se presentan diversas disyuntivas para cada persona y para la sociedad en general.

Para la explicación de estas contradicciones, la exposición se dividirá en tres partes a saber: la constitución ontológica de la formación humana, la formación para la preparación para el trabajo y la universidad y, por último, la formación académica.

\section{La constitución ontológica de la formación humana}

A partir del trabajo como categoría, el ser humano cambia constantemente en el acto de transformación de la naturaleza y se origina el proceso de producción, generando al mismo tiempo las condiciones necesarias para las bases históricas de la sociedad y de la colectividad. 
Esta constante transformación de la naturaleza, a través del trabajo por el ser humano, le permite su diferenciación con los animales, y su constitución histórica en las relaciones sociales, «por medio del trabajo, los hombres no sólo construyen materialmente la sociedad, sino también arrojan las bases para que se construyan como individuos. A partir del trabajo, el ser humano se hace diferente de la naturaleza, se hace un auténtico ser social, con leyes de desenvolvimiento histórico completamente distintas a las leyes que rigen los procesos naturales» (Lessa y Tonet 2008, 17). Este proceso conlleva la transformación del individuo en género humano y por tanto su constitución como sociedad, pues lo dota de conciencia y teleología.

El trabajo se constituye en la categoría fundante, ya que es a través de este que el individuo supera su base biológica y se transforma en un ser social, generando un salto cualitativo, y determinando la existencia humana. Es en la relación entre el ser humano y la naturaleza, a través del trabajo, que se produce la constitución de la conciencia, en el proceso de previa- ideación y objetivación ${ }^{2}$, en donde, el individuo crea las posibilidades y finalidades de su desarrollo histórico. Es así, que; «la esencia del trabajo consiste, justamente en la capacidad de rebasar la fijación del ser viviente en la relación biológica con su ambiente. El momento esencialmente distintivo no está dado por la perfección de los productos, sino por el papel de la conciencia» (Lukács 2004, 38). En síntesis el trabajo se constituye como el acto ontológico del ser social que genera formas de sociabilidad cada vez más complejas ya que «es una actividad de transformación de lo real por lo cual, el hombre se construye, concomitantemente, a sí mismo como individuo en la totalidad social, de la cual es partícipe» (Lessa 2002, 28). Es inherente al individuo, a través de las relaciones sociales que se expresan en su constitución y reproducción social,

El trabajo permite la constitución del ser social y su superación en términos biológicos. Por esto, el salto cualitativo permite la constitución del ser social, así como la

\footnotetext{
${ }^{2}$ Según Lessa y Tonet (2008), la previa-ideación y la objetivación son procesos que permiten al ser humano pensar sobre las diferentes alternativas para responder a una necesidad en el proceso de trabajo. Es el resultado de la anticipación de la conciencia hacia los fines probables de cada alternativa y su puesta en práctica. (c) (1) $\$(9$
} 
superación y transformación de la existencia social, es decir, de las relaciones humanas. Es así, que «con el trabajo se da al mismo tiempo -ontológicamente -la posibilidad de su desarrollo superior, del desarrollo de los hombres que trabajan. Es por esa razón- pero antes de todo porque se altera la adaptación pasiva, meramente reactiva, del proceso de reproducción del mundo circundante, porque ese mundo circundante es transformado de manera consciente y activa- que el trabajo se vuelve no simplemente un hecho en el cual se expresa la nueva peculiaridad del ser social, sino al contrario -precisamente en el plano ontológico-, se convierte en el modelo de la nueva forma del ser en su conjunto» (Lukács 2003, 135). Por esto la constitución social de la humanidad se establece como una constante transformación a partir de la relación entre el individuo y la naturaleza y del conocimiento que surge de esta.

Esto permite cada vez más la diferenciación entre el ser humano y los animales, es decir el proceso de humanización que «puede ser comprendido, pues, como la diferenciación y la complejización de las objetivaciones del ser social» (Netto y Braz 2011, 50). Esta transformación de las relaciones sociales produce el desenvolvimiento del ser social, por tanto,

sólo él [refiere al ser social] es capaz de actuar teleológicamente, sólo él se propone finalidades y anticipa metas- en suma, sólo él dispone de la capacidad de proyectar. ... En su acción y en su actuación, el ser social siempre encuentra alternativas y siempre puede escoger... el ser social dispone de la capacidad de sociabilización, esto es, él es responsable de la apropiación y desenvolvimiento por parte de los miembros de la sociedad en el interior de la propia sociedad, a través, fundamentalmente, de los procesos de interacción social, especialmente los educativos (formales e informales) (Netto y Braz 2011, 51-52).

El ser social, a través de la apropiación de la sociabilidad, logra aprender habilidades y conocimientos para su desarrollo histórico. Por tanto, uno de esos medios es la formación humana que, siguiendo a Tonet $(2012,77)$ es «el proceso del individuo singular cuando se torna miembro del género humano pasa por la necesaria apropiación del patrimonio -material y espiritual- acumulado por la humanidad en cada momento histórico».

\section{(c) (1) $\$(\Theta$}


La formación humana constituye, por parte del ser humano, un medio fundamental para la apropiación de conocimientos, para su constitución en las relaciones sociales expresadas en determinados momentos históricos de la sociedad y es la «articulación entre espíritu y materia, entre subjetividad y objetividad, entre interioridad y la exterioridad en el ser social» (Tonet 2012, 76). Es decir, es la forma en que materialmente el ser social se apropia de los conocimientos que surgen del proceso de transformación de la naturaleza, en su previa-ideación y objetivación; en otras palabras, del proceso de trabajo ${ }^{3}$.

La formación humana implica que, en el acto del trabajo, el ser humano no se apropie solo de conocimientos, sino también de otros elementos que le permitan su desenvolvimiento como persona y como parte de la sociedad. Por tanto, está relacionada con «un proyecto que entiende el desenvolvimiento material y subjetivo del ser humano como producto de una producción histórica y social» (Sudano 2008, 54). En este sentido, los conocimientos se convierten en universales como producto del desarrollo histórico y dialéctico de esta formación.

Mediante este acto de transformación surge la educación ${ }^{4}$ que tiene como finalidad «propiciar al individuo la apropiación de conocimientos, habilidades, valores, comportamientos, etc. que se constituyen en patrimonio acumulado y decantado a lo largo de la historia de la humanidad. De este modo, contribuye para que el individuo se construya como miembro del género humano y se torne apto para decidir hacer nuevas cosas de un modo que sean favorables para la reproducción del ser social en la forma que se presenta en un determinado momento histórico» (Tonet 2005, 142). Es decir, la formación humana es el

\footnotetext{
${ }^{3}$ Para Marx $(2010,192)$ el proceso de trabajo «es una actividad específica orientada a producir valores de uso, adecuar lo natural a las necesidades humanas, es la condición general del metabolismo entre el hombre y la naturaleza, la condición natural eterna de la vida humana, es, por tanto, independiente de cualquier forma de esta vida y más bien, común a todas sus formas sociales por igual». En este sentido, el trabajo como actividad personal del ser humano, contiene la materia prima y el medio de transformación por el cual actúa. Este proceso es el que permite la transformación constante del ser social.

${ }^{4}$ Expone Tonet (2005) que en el sentido ontológico la educación en sí misma no es trabajo, sin embargo, se constituye como una actividad humana. Asimismo, Sudano $(2008,54)$ señala que «la formación humana puede ser entendida también como proceso de educación de los sujetos».
}

\section{(c) (1) $(2)(-$}

Este obra está bajo una licencia de Creative Commons Reconocimiento-NoComercial-SinObraDerivada 4.0 Internacional. 
acto por el cual se apropian los conocimientos universales y al mismo tiempo la educación es la forma en que se transmiten históricamente, por esto entre ellas existe una relación dialéctica.

La formación y su expresión más concreta, la acción educativa, se constituyen en las formas con las que el ser social, se apropia, a través de la sociabilidad, del conocimiento humano generado a partir del proceso de trabajo. Además, es dirigida por la conciencia materializada en el proceso de trabajo. Es así como el ser humano se apropia de ese conocimiento que genera las condiciones materiales para la reproducción del género humano.

\section{La formación humana y su fractura en la sociedad capitalista}

Con la entrada del capitalismo, se produce la fractura del ser social, y la formación y la educación pasan a ser elementos determinantes para el proceso de producción del capital, $\mathrm{y}$, por ende, de la clase dominante en la conformación de sus intereses y su proyecto de sociedad. El trabajo se transforma de un proceso de creación de valores de uso a valores de cambio, lo que genera una sociabilidad basada en el capital. En esta lógica, el ser humano pierde su proceso consciente de creación y libertad, y se convierte únicamente en fuerza de trabajo. Por tanto «en esta forma de sociabilidad, la producción de mercancías -no importa bajo qué forma concreta- es un momento fundamental, entonces sí que sería razonable afirmar que la función hegemónica de la educación es la de preparar a los individuos para ser insertos en el mercado de trabajo. Pues, en esta forma de sociabilidad, el individuo vale en cuanto fuerza de trabajo y no en cuanto ser humano integral» (Tonet 2012, 16).

El capitalismo a través del capital transforma la función social de la formación humana y de la educación con la finalidad de que contribuyan a reproducir la sociabilidad necesaria para esta sociedad. Siguiendo a Tonet (2012), esa sociabilidad es contradictoria porque responde a intereses del capital y del trabajo. En esta lógica, la formación humana se configura como una preparación para el trabajo, que implica la reproducción de fuerza de trabajo para el capital, transformando al ser humano en mercancía necesaria para reproducir sus intereses y «la educación ocupa un lugar especial. Esto porque ella pasa a integrar cada (c) $(1) \Theta(\Theta)$

Este obra está bajo una licencia de Creative Commons Reconocimiento-NoComercial-SinObraDerivada 4.0 Internacional. 
vez más profundamente el proceso de producción. A partir de este momento, las clases trabajadoras no podrían recibir apenas una educación práctica para el trabajo» (Tonet 2005, 141). Entonces la adquisición de conocimientos universales se limita sólo a una parte mínima de la sociedad, lo que se genera como resultado la imposibilidad de pensar formas diferentes de sociabilidad a la que se conoce. El conocimiento obtenido es superficial y fragmentario lo que produce un colectivo de individuos alienados y deshistorizados.

La educación se convierte en un instrumento de las clases dominantes para la reproducir la alienación y para mantener la propiedad privada, en tanto «bajo el capital, es crucial asegurar que cada individuo adopte como propias las metas de reproducción objetivamente posibles del sistema. En otras palabras, en el sentido amplio del término educación, se trata de una cuestión de "internalización” por parte de los individuos ... de la legitimidad de la posición que les fue atribuida en la jerarquía social, junto con sus expectativas "adecuadas" y las formas "correctas" de conducta, estipuladas más o menos explícitamente en ese terreno» (Mészáros 2008, 40).

Sin embargo la formación humana y la educación, según Tonet (2012), inmersas en esta lógica generan una contradicción, ya que, si bien su función fundamental es la preparación de la fuerza de trabajo para el capital, se convierte, en un espacio donde la clase trabajadora puede apropiarse para la formación de conocimientos que impulsa la destrucción del capital. A partir de estos elementos, la formación académica en el marco de la universidad se expresa en un sentido contradictorio, pues, por un lado, posee como finalidad la preparación para el trabajo y, por otro lado, la posibilidad de generación de conocimientos universales y de su adquisición para la conformación de un proyecto de sociedad diferente.

\section{La universidad pública y la formación académica}

La Universidad, como centro de educación superior, está inserta en esta contradicción eminente, expresada entre la posibilidad de la autoconstrucción del ser humano, y la producción y apropiación del conocimiento por una clase determinada en la lógica de esta sociedad. En este sentido, Lessa $(2006,1)$ indica que «la relación entre la producción y la 
apropiación de conocimiento en una sociedad de clases es uno de los procesos sociales más complejos. La articulación entre el carácter genérico de la auto-construcción humana y las particularidades de las clases y grupos sociales hacen que los complejos envueltos en la producción y apropiación del saber sean influenciados por mediaciones casi infinitas».

Por tanto, la formación académica, la cual conlleva una lectura analítica de las condiciones materiales de la sociedad, está llena de contradicciones y refleja una serie de intereses contrapuestos y en disputa, principalmente expresados en la universidad pública. Es por esto que se convierte en un campo de intereses, tanto de la clase dominante como de la subalterna. Por un lado, es un marco socializador que coadyuva a la lógica del sistema, formando a personas para el mundo del trabajo; por otro lado, es un espacio de formación para la comprensión de la sociedad, de la producción y reproducción del conocimiento para la autoconstrucción del género humano, ya que «dentro de aquellos procesos de gran importancia para la formación de los sujetos, se puede situar los espacios de formación teórica, política y humana. Estos se tornan primordiales para ayudar en el develamiento de la explotación y de la dominación planteadas por el orden mercantil y como éste orden va afectando las diversas dimensiones de la vida de los sujetos» (Sudano 2008, 55).

Estos espacios académicos, posibilitan a las personas aspectos analíticos necesarios para comprender las formas en las que el capital se reproduce, y les permite poseer un pensamiento teórico y político crítico para superar el capitalismo. Es por esto, que la universidad se constituye como un espacio contradictorio; es decir, «una arena de contradicciones cruzada por la búsqueda de un proyecto hegemónico, por lo que su significado únicamente puede ser descifrado al concatenársele con el todo de las contradicciones de la totalidad social, y con las prioridades que define en ciertos momentos históricos de su desarrollo» (Esquivel 2008, 17).

La formación académica dentro de la universidad pública, se establece como un campo contradictorio pues, por un lado, se genera una formación que permite el desarrollo del ser humano y la apropiación del conocimiento; mientras que, por otro, se perfila únicamente en tanto genera mercancías para el capital. La formación académica universitaria (C) $\mathbb{D} \otimes \Theta$

Este obra está bajo una licencia de Creative Commons Reconocimiento-NoComercial-SinObraDerivada 4.0 Internacional. 
no se puede visualizarse como un elemento aislado de la reproducción de las relaciones sociales (de su fundamento ontológico); sino que se encuentra anclada a la totalidad social. Por ende, su lectura y análisis en la sociedad capitalista debe ser leída desde las transformaciones del trabajo, ya que encuentran mediaciones que se expresan en la reproducción del ser social. Debe tenerse en cuenta que, en la sociedad capitalista, «el ser social que logra ingresar a la educación superior es mediatizado por ella, por sus finalidades, sus bases racionales, su significado histórico en las relaciones sociales de producción y reproducción social, materializándose principalmente en la ubicación que ese ser social logre en la división social del trabajo» (Esquivel 2007,4).

En el marco del capital, la formación académica instaura las configuraciones necesarias para la reproducción de la fuerza de trabajo, a través de una educación para el mundo laboral, siendo este el espacio donde se constituyen las profesiones en el marco de la división social del trabajo. En síntesis, la formación académica se establece como un proceso contradictorio, que contiene y entrelaza una serie de elementos filosóficos, históricos, teóricos, metódicos, políticos, éticos, técnicos, culturales, ideológicos, y que se materializan en la lógica de reproducción de la sociedad.

La formación académica posee una serie de elementos que son contradictorios. Por un lado, está la posibilidad de generar conocimientos y lecturas críticas de la sociedad y por otro lado, da una formación para el mundo del trabajo, lo cual, no tiene que ver necesariamente con una escogencia de quien imparte esta formación, sino de las condiciones estructurales en que se desarrolla la sociedad. De esta manera, las posibilidades de la universidad pública son contradictorias, pero sientan algunas bases para la adquisición de conocimientos que puede permitir la lectura crítica y formativa de la sociedad desigual y excluyente en la que vivimos, de ahí su importancia como espacio en la construcción del género humano.

Las posibilidades de transformación de la formación académica, específicamente de la universidad, solo serán efectivas con la superación del capital, pero además el camino se debe dar en el sentido «de luchar por una universidad pública y gratuita, por su ampliación, (C) $\mathbb{D} \otimes \Theta$ 
por su memoria y por el acceso cada vez más amplio de la población a ella y luchar por los intereses de aquellos que realmente producen la riqueza (los trabajadores) pero no tienen acceso a ella» (Tonet 2012, 93). Es decir, aun cuando sé es consciente de las contradicciones que sufre constantemente la universidad pública, debe notarse que es uno de los espacios de adquisición de conocimientos universales y de la comprensión de la sociedad, por esto es importante desvendar sus paradojas pero con la finalidad de fortalecer el pensamiento crítico para la lectura de la sociedad contemporánea.

\section{Conclusiones}

Las contradicciones que se presentan en el capitalismo en las que se basa la sociedad contemporánea remite constantemente a entender la relación entre el trabajo y el capital y en ese sentido, todas las formas de sociabilidad y reproducción del género humano se encuentran inmersas en esta lógica. La formación humana y la educación no están exentas, por esto su función de proveer conocimientos universales que le permitan al género humano su desenvolvimiento se ve trastocada por la preparación para el trabajo.

La formación humana y la educación son mediaciones para la transmisión del conocimiento al género humano, por eso es importante develar su base ontológica para entender sus contradicciones en el desarrollo de la sociedad capitalista y los cambios sufridos en su función social a partir del capital. Los conocimientos universales que permiten entender la totalidad social, en esta lógica son atomizados y posiblemente inaccesibles para una parte mayoritaria de la sociedad, lo que limita no sólo el pensamiento crítico sino la comprensión histórica del género humano. Por lo que, repensar las contradicciones que sufre la educación y principalmente en las universidades públicas permite discutir sus posibilidades y retomar la importancia de estas en este momento histórico.

El paso de una formación humana a una académica permite comprender las posibilidades reales entre una educación que permita la emancipación humana o una que tenga como función la preparación en función de un mercado de trabajo. Es en esta (c) $\frac{10(9)}{\mathrm{BY} N \mathrm{NC}}$

Este obra está bajo una licencia de Creative Commons Reconocimiento-NoComercial-SinObraDerivada 4.0 Internacional. 
contradicción que se desvela la necesidad de que las universidades públicas comprendan su papel de generación de conocimientos colectivos para el género humano, en tanto, no se limite a conocimientos únicamente técnicos, prácticos o profesionales sino que su centralidad sea la de formar seres humanos libres y pensantes. La universidad, como espacio contradictorio, debería proveer todo el conocimiento humano que permita a las personas entender su desarrollo como parte del género humano, así como entender las posibilidades y contradicciones que sufren principalmente en la sociedad contemporánea desigual y excluyente.

Como reflexión final, entonces es importante cuestionar y reflexionar sobre nuestra posición como docentes pero también se deben entender las condiciones estructurales en las que se desenvuelve la sociedad, en tanto, es necesario comprender la contradicción para posicionarnos frente a ella. Siendo la educación un medio para propiciar un debate ante la desigualdad y exclusión, y frente a postulados cada vez más conservadores, que limitan la posibilidad de pensamiento crítico.

\section{Referencias}

Esquivel Corella, Freddy. 2007. «Análisis de las contribuciones entre la formación académica de la Universidad de Costa Rica y las bases orgánicas institucionales que la vinculan con el tema de pobreza». Tesis de Doctorado en Educación, Universidad Estatal a Distancia.

. 2008. «Acercamientos a la valoración de la formación académica de la Universidad de Costa Rica y su vínculo con la realidad nacional». Revista Educación 32, 13-28.

Lessa, Sergio. 2002. Mundo dos homens. Trabalho e ser social. São Paulo, Brasil: Boitempo Editorial.

. 2006. «Universidade: produção e apropriação do conhecimento». Manuscrito inédito. http://www.sergiolessa.com/no_published/universidade_conhecimento.pdf.

Lessa, Sergio e Ivo Tonet. 2008. Introdução a filosofia de Marx. São Paulo, Brasil: Editora Expressão Popular.

\section{(c) (1) $(9)$}


Lukács, Georg. 2003. «Las bases ontológicas de la actividad humana». En Servicio Social Crítico. Hacia la construcción del nuevo proyecto ético-político profesional, organizado por Elisabete Borgianni, Yolanda Guerra y Carlos Montaño, 129-152. São Paulo. Brasil: Cortez Editora.

2004. Ontología del ser social El Trabajo. Argentina: Ediciones Herramienta.

Marx, Karl. 2010. El Capital. Crítica de la economía política. Tomo I. Libro 1. Proceso de producción del capital. Santiago, Chile: LOM Ediciones.

Mészáros, István. 2002. Para Além do Capital. Rumo a uma teoria da transição. São Paulo. Brasil: Editorial Boitempo.

. 2008. La educación más allá del capital. Argentina: Siglo veintiuno editores.

Netto, José Paulo y Marcelo Braz. 2011. Economia Política. Uma introdução crítica. São Paulo. Brasil: Cortez Editora.

Sudano, Suliane. 2008. «Subjetividades em movimento: movimentos sociais e processos de formação humana». Tesis de Maestría, Universidade Federal do Rio de Janeiro. https://sites.google.com/site/secretariappgss/banco-de-teses-e-dissertacoes-do-ppgss

Tonet, Ivo. 2012. Educação contra o capital. São Paulo. Brasil: Instituto Lukács. 2005. Educação, Cidadania e Emancipação Humana. São Paulo. Brasil: EDUFAL. http://ivotonet.xp3.biz/arquivos/EDUCACAO_CIDADANIA_E_EMANCIPACAO _HUMANA.pdf 